\title{
Notizen zur Klassenanalyse der BRD durch das PKA (2)
}

In der gegenwärtigen marxistischen Diskussion der Klassenanalyse Westdeutschlands sind im wesentlichen zwei Richtungen zu unterscheiden (3). Die eine macht sich daran, den wirklichen Akkumulationsprozeß des gesellschaftlichen Gesamtkapitals und seiner Teile, die Bedingungen und Formen staatlicher Tätigkeit, die daraus folgenden Lebensbedingungen der Lohnarbeiter zu untersuchen und knüpft daran erste Analysen von Bewußtseinsformen und Kämpfen als der Grundlage jeder revolutionären Taktik. $\mathrm{Zu}$ dieser Richtung wären etwa viele Arbeiten im Rahmen dieser Zeitschrift zu zählen. Die andere Richtung bemüht sich zunächst darum, die erscheinende Sozialstruktur mithilfe der aus der bürgerlichen Produktionsform sich ergebenden Klassenbestimmungen zu entschlüsseln und sozialstatistisch zu ordnen. Hierher gehören etwa die Arbeiten des IMSF und die hier zur Debatte stehende Untersuchung des Projekts Klassenanalyse (PKA). Die Arbeiten des Instituts für marxistische Studien und Forschungen (IMSF) leiden infolge der Unverbundenheit und des Zusammenwerfens von Momenten des Arbeitsprozesses, der Wertbewegung und der realen Geschichte an erheblichen analytischen Fehlern und Unschärfen (4). Demgegenüber stellt die Untersuchung des PKA im Rahmen des sozialstatistischen Zweigs der Klassenanalyse zweifellos eine fortgeschrittenere Form dar. In der gründlichen statistischen Aufarbeitung der Klassenstruktur ist ein sehr wichtiger Beitrag zur gegenwärtigen Klassenanalyse zu sehen.

Wenn die Aufgabe der weiteren klassenanalytischen Arbeiten sicher darin bestehen wird, die Widersprüche zwischen ihren Ansätzen (5) im Fortgang des

1 Gerhard Armanski, Norbert Kostede, Boris Penth, Jörg Pohlmann. Den wissenschaftlichen und politischen Arbeitsplan des Redaktionskollektivs werden wir im nächsten Heft der Prokla vorlegen.

2 Projekt Klassenanalyse: Materialien zur Klassenstruktur der BRD. Zweiter Teil. Grundriss der Klassenverhältnisse (1950-1970). Berlin (W) 1974. Auf den ersten Teil der Materialien werden wir nur da eingehen, wo es von der Kritik des zweiten Teils her erforderlich ist.

3 Von der Klassenanalyse kommunistischer und sozialistischer Gruppen und Parteien sehen wir hier ebenso ab, wie von diversen auf dem Phänomen des ,Massenarbeiters" beruhenden Arbeiten. Sie müssen späteren Untersuchungen vorbehalten bleiben. Vgl. die partielle Kritik durch Gerhard Armanski, Staatliche Lohnarbeiter im Kapitalismus, Probleme des Klassenkampfs 16/1975, S. 6 ff.

5 Diese Widersprüche liegen im wesentlichen nicht in den beteiligten Personen begründet, sondern in den Wirkungen der partiellen Entfaltung der Klassenkämpfe, dem Nachhall der Studentenbewegung, dem zersplitterten Bildungsprozeß der revolutionären Linken, weiter in den realen strukturellen Umwälzungen des Klassengefüges und schließlich in scheinbaren und wirklichen Widersprïchen der Marx'schen Klassentheorie. 
Untersuchungsprozesses aufzuheben, muß die Frage gestellt werden, wie das geschehen soll. Das verweist auf das Problem des richtigen Ausgangspunktes und Fortgangs. Wenn als Leitfrage der Klassenanalyse gelten soll, daß sie die gegenwärtig und zukünftig praktisch zur Umwälzung der bürgerlichen Gesellschaft drängenden und befähigten sozialen Kräfte aufdecken soll, dann muß auch im jetzigen Stadium der Diskussion gezeigt werden, ob die einzelnen Ansätze dazu in der Lage sind. Mit dieser Absicht wollen wir die vorliegende Untersuchung des PKA einer ersten kritischen Prüfung unterziehen (6).

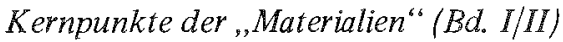

Folgt man den einzelnen Argumentationsstufen des PKA in ihrer Untersuchung der Klassenstruktur der BRD, so lassen sich fünf Schwerpunkte ausmachen:

1. Gemäß ihrer zentralen methodischen Prämisse, die Darstellung der Klassen und der ökonomischen Bedingungen des Klassenkampfs habe von den Formen auf der Oberfläche der bürgerlichen Gesellschaft auszugehen, wie Marx sie im letzten Abschnitt des dritten „Kapital"-Bandes analysierte, begreift das PKA zunächst als allgemeinen Typus der Klassenstruktur die Existenz der Lohnarbeiterklasse, der Kapitalistenklasse und der Klasse der Grundeigentümer (6a). Nun erkennen die Autoren in Anlehnung an entsprechende Marx'sche Ausführungen, daß die Grundeigentümer mit Notwendigkeit im Voranschreiten der kapitalistischen Produktionsweise an gesellschaftlicher Bedeutung verlieren, weshalb in ihrer weiteren Analyse der herrschenden Klassenstrukturen und Klassenverhältnisse im Grunde nur zwei Hauptklassen von Relevanz bleiben, die (produktiven und kommerziellen) Lohnarbeiter des Kapitals und die Kapitalistenklasse.

2. Zum einen die von Marx zwar beabsichtigte, aber nicht vollzogene Ableitung des bürgerlichen Staates, zum anderen die immer offenkundigere Bedeutung der unproduktiven und inbesondere staatlichen Lohnarbeiter im Klassengefüge der modernen Gesellschaft führen das PKA zu der Einsicht, daß die Entwicklung eines allgemeinen Typus der Klassenstruktur den Begriff der staatlich-politischen Formen der bürgerlichen Gesellschaft zur Voraussetzung hat:

„Die spezifischen Beziehungen des Staates zur Gesellschaft müssen entwickelt

6 Wir sind uns darüber im klaren, daß die Fülle des vom PKA vorgelegten Materials damit noch nicht hinreichend verarbeitet ist. Unser Verfahren erscheint uns aber gerechtfertigt, um zentrale Momente der Klassenanalyse nicht aus dem laufenden Diskussionsprozeß verschwinden $\mathrm{zu}$ lassen.

$6 \mathrm{a}$ „Materialien ..." Bd. I, S. 19; es muß hier vermerkt werden, daß im Redaktionskollektiv Klassenanalyse unterschiedliche Auffassungen hinsichtlich dieser methodischen Prämisse vertreten werden; insbesonder ist umstritten, ob die Kategorie der Lohnarbeit, wie sie auf dieser Ebene erscheint, als Ausgangspunk t für die Analyse der realen gesellschaftlichen Lage und des Bewußtseins der Arbeiterklasse begriffen werden kann. Daraus ergibt sich für die Bestimmung der Lohnarbeiter die Frage, ob diese als Lohnarbeiterklasse oder als Arbeiterklasse und lohnarbeitende Mittelklasse zu fassen sind. 
und seine einzelnen Funktionen charakterisiert werden, um so die besonderen Bestimmungen der von ihm beschäftigten Lohnarbeiter zu erhalten - der wichtigste Schritt zur Lösung des Problems der Verallgemeinerung der Lohnarbeit" (7).

Jedoch wird dieser Bereich der Lohnarbeiter nicht in den Begriff der Arbeiterklasse einbezogen, vielmehr führen die Autoren auf dieser Ebene der Darstellung den Marx'schen Begriff der Mittelklasse ein: insbesondere die staatlichen Lohnarbeiter, die ,Staatsagenten" (PKA), müssen als ein Bereich der Mittelklasse begriffen werden, auch wenn dieser Bereich strikt von den, traditionellen" Bereichen der Mittelklasse (Kleinbürgertum, Handwerker etc.) un terschieden wird.

3. Es charakterisiert die Schriften des PKA, daß die Erarbeitung eines allgemeinen Typus der Klassenstruktur zurecht immer die Frage einschließt, welche Möglichkeiten der Herausbildung von gesellschaftlichem Bewußtsein und insbesondere von Klassenbewußtsein für die unterschiedlichen Klassen, Fraktionen und Schichten bestehen. Doch kann die Frage nach den allgemeinen Konstitutionsbedingungen gesellschaftlichen Bewußtseins nicht mit der Frage nach der Bildung des realen historischen Bewußtseins vermittelt werden. Da aber die Autoren diese Vermittlungsschritte nicht vollziehen, ist es nur konsequent, wenn sie in ihrer Analyse der Klassenverhältnisse in der BRD „keine genauere Untersuchung der Bewußtseinsformen von Klassen und Klassenfraktionen" (8) vornehmen können und diesen für eine Realanalyse doch wesentlichen Aspekt fallen lassen müssen, bzw. sich in aufgesetzten allgemeinen Leerformeln erschöpfen.

4. In einer umfangreichen und detaillierten Analyse statistischen Materials (Bevölkerungs-, Beschäftigungs-, Einkommensstatistik etc.) versucht das PKA im zweiten Band der ,Materialien“" die Klassenverhältnisse der BRD aufzuschlüsseln. Ausgehend von ihrer Darstellung eines allgemeinen Typus der Klassengliederung beabsichtigen die Autoren die "Untersuchung eines konkreten Stuicks Zeitgeschichte" (PKA), wobei es ihnen offenbar um eine ,quantitative Weiterentwicklung des Marxismus $^{66}$ geht, denn sie führen aus, daß ,alle Konzeptionen, die von einer, qualitativen Weiterentwicklung des Marxismus im Verlauf der geschichtlichen Entwicklung der bürgerlichen Gesellschaft ausgehen, weder zu einer zusammenhängenden und konsequenten revolutionären Taktik gelangen, noch den widersprüchlichen Prozeß der Entwicklung der eigenen Bewußtseinsformen in der jüngsten Vergangenheit erklären“ können (9).

5. In einem letzten Schritt versuchen die Autoren aus ihrer gesamten Untersuchung die „Grundzüge einer revolutionären Taktik“ zu entwickeln, wobei im Mittelpunkt die Frage der Mittelklasse steht: zwar begreift das PKA insbesondere die staatlichen Lohnarbeiter als Mittelklasse und nicht als Teil der Arbeiterklasse, doch führt es aus, daß die Taktik der Arbeiterbewegung gegenüber den „Staatsagenten“ als eine „Aktionseinheit“ entwickelt werden müsse. Auch wenn die Genossen hier recht

7 ebenda Bd. I, S. 16

8 ebenda Bd. II, S. 13

9 ebenda, von uns hervorgehoben 
konfuse und widersprüchliche Formulierungen gebrauchen (,Aktionseinheitspolitik", ,Aktionsbündnis" etc.), so halten sie doch letztlich eine „Bündnispolitik (für) das verfehlte "Mittel" (10), um die staatlichen Lohnarbeiter in den Kampf der Arbeiterbewegung einzubeziehen.

\section{Zum Verhältnis von Struktur und Dynamik der Klassengliederung}

Einer der zentralen Mängel der Klassenanalyse des PKA besteht in der weitgehenden Ausblendung der Dynamik der Klassengliederung, der innere Motor dieses Gliederungsprozesses bleibt unbegriffen. Wir müssen hier keinen Maßstab von außen an die Arbeit des PKA herantragen, vielmehr messen wir die Autoren an ihrem eigenen Anspruch:

„Die Darstellung der Entwicklungsgesetze (der) ökonomischen Basis ist die Voraussetzung für die Analyse der empirisch gegebenen Vielfalt der Erscheinungen in der Bevölkerungsstruktur eines Landes" (11).

Nimmt man dieses Motto ernst, so hätte das PKA den zweiten Band der „Materialien“ noch gar nicht schreiben dürfen, denn vor der Analyse der Bevölkerungsstruktur der BRD hätten die Autoren ihrem Anspruch zufolge die „Entwicklungsgesetze der ökonomischen Basis" zur Darstellung bringen müssen, also insbesondere die Gesetzmäßigkeiten der Kapitalakkumulation als die den Prozeß der Klassenstrukturierung dominierenden Gesetze. Doch weder wagt man sich an eine Diskussion der logischen Struktur der Marx'schen Akkumulations- und Krisentheorie heran, die ja nicht gerade von unmittelbar einleuchtender Eindeutigkeit und Stringenz geprägt ist, noch hat man die bisherigen kontroversen Diskussionen auf diesem Gebiet herangezogen, insbesondere die Diskussion zur Theorie des Profitratenfalls und seiner Bedeutung für die zyklische Bewegungsform des Kapitals. Allein dies hätte die Autoren in die Lage versetzt, die Vermittlungen von ökonomischer Entwicklung, Klassenstrukturierung und Bewußtseinsformen aufzudecken.

Doch bleibt selbst die in diesem Motto unterstellte Reihenfolge zu kritisieren: erst die Darstellung des allgemeinen Typus der Klassengliederung - dann die Darstellung der ökonomischen Entwicklungsgesetze - dann die Analyse der realen Bevölkerungsstruktur. Die materialistische Klassentheorie hat von Beginn an den Zusammenhang von Struktur und Dynamik der Klassengliederung zu reflektieren, nicht erst im Übergang zur Analyse ,eines konkreten Stücks Zeitgeschichte“. Wie uns die Ursachen und die Durchsetzung der marginalen Entwicklung der Grundeigentümerklasse ein Rätsel bleiben, wie die historisch sich durchsetzende radikale Umwälzung des privaten Dienstleistungssektors ein Rätsel bleibt, so bleibt auch unbegriffen, in welchen Verhältnissen die gesellschaftliche Gesarntarbeit auf die unterschiedlichen Sphären von Produktion und Reproduktion aufgeteilt werden

10 ebenda Bd. II, S. 443

11 ebenda Bd. I, S. 13; von uns hervorgehoben 
kann und muß, wenn wir von denjenigen Bewegungen abstrahieren, die diese Strukturierungsprozesse dominieren, nämlich den Bewegungen der Kapitalakkumulation. Wer also die „Klassengliederung ihrem allgemeinen Typus nach“ (PKA) entwickeln will, kann nicht beim bloßen Nachvollzug der inneren Strukturen des modernen Klassengefüges stehenbleiben, sondern der muß zugleich die komplexen Prozesse darstellen, in welche die Klassengliederung einge bettet ist.

Hier scheint die zentrale methodische Prämisse des PKA, die Darstellung der Klassen und der ökonomischen Bedingungen des Klassenkampfs habe die Formen auf , der ${ }^{6}$ Oberfläche der bürgerlichen Gesellschaft zum Ausgangspunkt, in eine ,methodische Sackgasse ${ }^{6}$ zu führen. Sie hat beim PKA zur Folge, die dieser verknöcherten Oberfläche zugrundeliegenden sozialen Beziehungen und ökonomischen Prozesse aus dem Auge zu verlieren.

Wenn nun das PKA in seinem zweiten Band der ,Materialien“ den realen Prozeß der Klassengliederung in der BRD verfolgt, so bleibt den Autoren auf Grund mangelnder Entwicklung der spezifischen Dynamik der Klassengliederung nichts anderes übrig, als den Prozeß der realen Klassenstrukturierung über statistische Arbeitsweisen zu verfolgen, als den Prozeß der ,Klassengliederung in den Sozialstatistiken aufzuspüren" (12). Sicherlich erfordert die Untersuchung der realen Klassenstrukturen und Klassenverhältnisse die Aufarbeitung statistischen Materials und hier liegt das eigentliche Verdienst des PKA -, doch weder stellt dies das Zentrum der Untersuchung der wirklichen Klassenverhältnisse der (Klassenanalyse ist weit mehr als materialistisches Fliegenbeinezählen!), noch kann die Aufarbeitung dieses Materials losgelöst von der Untersuchung der politisch-ökonomischen Prozesse erfolgen, deren klassenstrukturelle Reflexe sich dann - in welch verquerer Form auch immer - in den bürgerlichen Sozialstatistiken niederschlagen. Wer die Bewegungen der einzelnen Klassen und Fraktionen nicht allein als „empirische Kovarianzen" (etwa: Bewegungsentsprechungen) zwischen industriellem Zyklus und Verschiebungen in der Bevölkerungsstruktur ,,verzeichnen" will, wer vielmehr die Entwicklungsrichtung dieser Strukturverschiebungen und deren Notwendigkeit (!) erkennen will, der muß diese inneren Bewegungen im herrschenden Klassengefüge zunächst einmal aus den inneren Gesetzmäßigkeiten und dem historischen Verlauf der Akkumulation ableiten. (12a).

Die 16seitige (!) Analyse der wirtschaftlichen Entwicklung in der BRD von 1950 bis 1970 (13) verbleibt notwendigerweise auf einer deskriptiven Ebene, die erscheinende Bewegung des BRD-Kapitals und die Zyklizität dieser Bewegung können nicht auf ihre konstitutiven Gesetzmäßigkeiten befragt werden, da man weder die logische Struktur des Akkumulations- und Krisenprozesses entwickelt hat noch danach fragen könnte, in welcher Weise sich diese Gesetzmäßigkeiten in der realen wirtschaftlichen Entwicklung durchsetzen. Und so nimmt es auch nicht

12 ebenda Bd. II, S. 244

12a Vgl. dazu Norbert Kostede, Akkumulation und Mittelklassen, in: Probleme des Klassenkampfs $13 / 1974$

13 ebenda Bd. II, S. $47 \mathrm{ff}$ 
wunder, wenn das PKA die Wirkungen der Akkumulation des BRD-Kapitals auf die konkreten Reproduktionsbedingungen der unterschiedlichen Bereiche der Arbeitsbevölkerung häufig außerhalb der Betrachtung lassen muß: „Dies sind alles Aspekte, die uns im Rahmen dieser Arbeit nicht interessieren" (14). Wie kann man aber dann noch den Anspruch aufrechterhalten, über einen „Grundriß der Klassenverhältnisse" die „Grundzüge der revolutionären Taktik in der BRD" zu entwickeln?

Wenn hier die Kritik vorgetragen wird, daß die Dynamik und der innere Motor der Klassengliederung weitgehend aus der Untersuchung des PKA herausfällt, so umfaßt diese Kritik zwei weitere Aspekte, zum einen die Frage nach der Entwicklung der realen Arbeitsprozesse, zum anderen die Frage nach den politischen Prozessen, die in die Klassengliederung hineinspielen. Zunächst zu ersterem. Die Autoren führen aus:

„Wollte man die innere Struktur des Gesamtarbeiters ... allein oder zuerst aus dem stofflichen Inhalt der verschiedenen Tätigkeiten ableiten, wären nur willkürliche Zusammenfassungen einzelner Gruppen zu besonderen Fraktionen möglich. Bei der Untersuchung der konkreten Gestalt einer Gesellschaft könnten nur die Bewegungen erklärt werden, die sich aus den veränderten stofflichen Bedingungen des Arbeitsprozesses in der Fabrik ergeben. Hiermit aber wäre weder über die bestimmte innere ökonomische Struktur des Gesamtarbeiters dieser Gesellschaft noch etwas über den Grad der gesellschaftlichen Antagonismen ausgesagt ${ }^{66}(15)$.

Als würde z. B. die immer offenkundigere Arbeitsteilung in Kopfarbeiter und Handarbeiter, wie sie auch im materiellen Arbeitsprozeß zum Ausdruck kommt, nichts über den "Grad der gesellschaftlichen Antagonismen" aussagen! Die mangelnde Reflexion der gesellschaftlichen Rolle der Intelligenz in der bürgerlichen Gesellschaft (auf 1000 Seiten hätte doch ein kleines Abschnittchen über diese für die materialistische Klassenanalyse doch wesentliche Fragen drin sein müssen!) ist nur ein Beispiel dafür, daß alles, was nicht unmittelbar aus den Formbestimmungen der gesellschaftlichen Arbeitsprozesse abgeleitet werden kann, ,unter die Räder ${ }^{6}$ zu geraten droht. Sicherlich haben die Autoren recht, wenn sie als Ausgangspunkt ihrer Untersuchung der Struktur des Gesamtarbeiters die spezifischen Formen analysieren, in denen menschliche Arbeit im Reproduktionsprozeß der bürgerlichen Gesellschaft wirkt (produktive, kommerzielle, unproduktive Arbeit etc.). Dennoch bleibt zu kritisieren, daß die konkrete Gestalt der industriellen und kommerziellen Arbeitsprozesse und der privaten und staatlichen Dienstleistungsprozesse sowie deren historische Umwälzungen weitgehend aus der Untersuchung herausfallen und folglich auch nicht in ihrer Bedeutung für die Strukturierung des Klassengefüges erkannt werden können. Die Arbeit des PKA stellt gewissermaßen die Kehrseite der modernen Industrisoziologie dar, die auf Grund ihrer Indifferenz gegenüber den spezifischen Formen des gesellschaftlichen Arbeitsprozesses in der begriffslosen Analyse von Technologie und Arbeitsmethodik verharrt, während umgekehrt das PKA die 
historischen Entwicklungen von Technologie und Arbeitsmethodik vollkommen ignoriert und sich in einer sterilen Formanalyse erschöpft. Wer Struktur und Dynamik der herrschenden Klassengliederung einholen will, der muß auch diese.Stufe der Untersuchung einbeziehen. (Wir werden im folgenden noch darauf zurickkommen, wie sich ihre Indifferenz gegenüber dem realen Arbeitsprozeß und seinen historischen Entwicklungen in ihren Ausfuihrungen zum Bereich der staatlichen Lohnarbeiter rächt.)

Es ist offenkundig, daß nicht allein ökonomische, sondern zugleich politische Prozesse auf die Klassengliederung einwirken. Wenn sich die Autoren des PKA nun die Frage stellen, warum es zur Aufblähung der staatlichen Lohnarbeiterschichten kommt, so führen sie hinsichtlich der repressiven Funktionen des Staatsapparates aus:

„Der bürgerliche Staat ist einerseits wesentlich Produkt des Klassenantagonismus. Er hat daher eine Reihe von Funktionen wahrzunehmen, die unmittelbar aus dem spezifischen Charakter der bürgerlichen Produktionsweise entspringen und im Kern der Aufrechterhaltung dieser sozialen Verhältnisse dienen ... Eine notwendige Folge der Abwehr und Eindämmung dieser Bedrohung (der bürgerlichen Ordnung durch die Zuspitzung der sozialen Konflikte und Antagonismen; Red. koll.) besteht im Ausbau der staatlichen Machtmittel, also auch in der personellen Verstärkung des Staatsapparates" (16).

Doch mit einer derartigen Begründung der repressiven Funktionen des bürgerlichen Staates ist es nicht getan, wenn man begreifen will, warum die repressiven Staatsagenten mit Notwendigkeit an Umfang und Bedeutung im Klassengefüge der BRD gewonnen haben, hier hätte man den politischen Prozeß analysieren müssen, der diese Funktionen zur Durchsetzung kommen läßt: den realen Verlauf des Klassenkampfs. Aus der Analyse der politisch-staatlichen Reaktionen auf die Entwicklung der Klassenkämpfe in der BRD, aus der Analyse der Entwicklung der politischen und militärischen Aufgaben der BRD in der europäischen Sphäre etc. hätte man ableiten können, welche Notwendigkeiten für die Ausdehnung dieses Sektors der lohnarbeitenden Mittelklasse bestanden haben. Ohne diesen Schritt der Vermittlung von politischem Prozeß und Klassengliederung bleibt man jedoch wiederum darauf verwiesen, diese Entwicklung der realen Klassenstruktur anhand einer sozialstatistischen Analyse zu „verzeichnen".

Resümieren wir und treiben wir den bisherigen Gang unserer Kritik auf die Spitze: Mit ihrer Vorstellung, es ginge bei der materialistischen Klassenanalyse des modernen Kapitalismus um so etwas wie eine „quantitative Weiterentwicklung des Marxismus" sitzen die Autoren des PKA selbst noch dem Mystizismus von der ,geschichtslosen ${ }^{6}$ Oberfläche der bürgerlichen Gesellschaft auf. Diese Vorstellung wurzelt in ihrer Begrifflosigkeit gegenüber der komplexen Dynamik des herrschenden Klassengefüges. Das Kapital als sich ständig selbst revolutionierendes gesellschaftliches Verhältnis verändert die differenzierten Strukturen seines jeweils histo- 
risch-konkreten Klassengefüges: auch wenn dieses Klassengefüge im Fortschritt der herrschenden Produktionsweise immer deutlicher die dominierenden und zentralen Klassenstruturen zur Ausprägung brachte, so erfuhr es doch zugleich bedeutsame strukturelle Umwälzungen. Unsere Aufgabe besteht nicht darin, diese strukturellen Umwälzungen zu leugnen und die Analyse der heutigen Klassenstrukturen auf die simple Frage nach Quantitäten zu reduzieren, unsere Aufgabe besteht vielmehr darin, die historischen Umwälzungen des Klassengefüges aus der inneren Natur und Dynamik des Kapitals selbst zu erklären.

\section{Einzelpunkte der Kritik (17)}

Auch wenn wir der Untersuchung des PKA im einzelnen folgen, sie sozusagen immanent kritisieren, lassen sich Widersprüche in der ,Zurechnung“" von Bevölkerungsteilen aufzeigen, die zum Teil aus begrifflichen Unschärfen, zum Teil aus der fast vollkommenen Ausblendung der historischen Wirklichkeit sich ergeben.

Um das staatliche Personal näher zu untergliedern, unterscheidet das PKA „bei den Staatsfunktionen drei große Bereiche:

A. Funktionen, die der bürgerlichen Form der Arbeit entspringen;

B. Funktionen, die sowoh! der bürgerlichen Form als auch der Entwicklung der gesellschaftlichen Potenzen der Arbeit entspringen;

C. Funktionen, die dem gesellschaftlichen Charakter der Arbeit entspringen" (18) Komplex A wird als „zentrale Regierungsmaschinerie“", Komplex B als ,Übergangsbereich"s gefaßt.

So einleuchtend diese Einteilung auf den ersten Blick erscheint, sowenig hilft sie letztlich weiter und wirft mehr analytische und praktische Fragen auf, als es sie löst. Die Unterscheidung ist zunächst fragwürdig, weil mit ihr der Zusammenhang von bürgerlicher Produktionsform und Vergesellschaftung der Arbeit auseinandergerissen wird. Dieses Vorgehen auf den produktiven Gesamtarbeiter angewandt, könnte etwa die Abteilungen der Produktionsplanung des Kapitals dem ,gesellschaftlichen Charakter der Arbeit" zurechnen, Meister, Vorarbeiter und Fertigungsingenieure der ,bürgerlichen Form der Arbeit", käme aber bezüglich des eigentlichen produktiven Arbeitspersonals in große Schwierigkeiten. Denn die Kombination der Arbeiter in der großen Industrie ist zugleich der bürgerlichen Form der Arbeit und in dieser auch der Entwicklung ihrer gesellschaftlichen Tendenzen geschuldet. Ähnliche Probleme stellen sich bezïglich des Staatsektors, wo sich zeigen läßt, daß die genannte Unterscheidung meist nicht durchgehalten werden kann, weil sich die Funktionen überschneiden.

17 Wir beschränken uns hier auf Fragen, welche die staatlichen Lohnarbeiter und die Mittelklasse betreffen. Andere, etwa die uns sehr problematisch erscheinende Bestimmung der Minimalgröße des Kapitals (wo das konstante Kapital herausfällt; der ausschließliche Bezug aufs Handwerk) Bd. II/S. 230 ff., müssen leider beiseite bleiben. 
Ist etwa die zum Bereich „öffentliche Sicherheit und Ordnung“ zählende Bauund Gesundheitspolizei nur der bürgerlichen Form der Arbeit geschuldet? Oder das Nachrichten- und Wohnungswesen nur dem gesellschaftlichen Charakter der Arbeit? Vielmehr dürften, von wenigen eindeutigen Extremen auf beiden Seiten abgesehen, die meisten staatlichen Funktionen doppelt bestimmt sein. ,Sie entspringen der Entfaltung der Produktivkräfte der Arbeit, sind aber wesentlich bestimmt von dem spezifischen Charakter der Produktionsverhältnisse“ (19). Auch für den Staat gilt, daß sich im Kapitalismus der gesellschaftliche Charakter der Arbeit nur im Rahmen und vermittels seiner bürgerlichen Form durchsetzt, daß der Inhalt der sozialen Revolution nicht etwa im Aufstand der ,,nützlichen ${ }^{65}$ gesellschaftlichen gegen die „unnützen“ bürgerlichen Kräfte besteht, sondern in der gewaltsamen Abstreifung der bürgerlichen Produktionsform, der alle Mitglieder der bürgerlichen Gesellschaft mehr oder minder unterliegen (20).

Die noch im 1. Band der „Materialien..." entwickelten Bestimmungen zu Form und Produktion des bürgerlichen Staates verflüchtigen sich in der konkreten Analyse zu einer Koppelung von abstrakter Formbestimmung und begriffslosen Oberflächenkriterien. Das was zu leisten ist bei der Bestimmung der staatlichen Lohnarbeiter, die genaue allgemeine und konkrete Untersuchung ihrer Stellung in der ökonomischen und politischen Reproduktion der bürgerlichen Gesellschaft, die exakte Herausarbeitung der Staatsfunktionen also, fällt bei einer Konstruktion mitten hindurch.

Die Analyse gerät vollends ins Schwimmen, wenn die zunächst zum Übergangsbereich gezählten Träger der Bildung und Ausbildung, die Lehrer und Wissenschaftler, plötzlich als Hauptmasse des, ,geistigen Unterdrückungsapparates ${ }^{66}$ auftauchen (21). Zu diesem gehören also nun Nuklearröntgenologen in den Universitätskliniken, Physiker und Chemiker in den Universitäten, Biologielehrer an den Schulen ebenso wie Volkswirtschaftslehredozenten und Sozialkundelehrer. Wenn das eingeschlagene Zurechnungsverfahren vielleicht bei den letzten noch angehen mag, so ist es insgesamt zur analytischen Erfassung dieses Teils des Staatspersonals vollkommen untauglich.

Ähnliche Probleme tauchen auf, wenn die medizinische und soziale Dienste leistenden ,aktiven Staatsagenten ${ }^{66}$ keiner weiteren Untersuchung gewürdigt werden, sie konsequent auch im Abschnitt über revolutionäre Taktik nicht mehr auftauchen. Sind die Beschäftigten der öffentlich-rechtlichen Banken und Kreditinstitute $\mathrm{zu}$ den Staatsbeschäftigten zu rechnen (22), oder sind sie nicht vielmehr zum großen Teil kommerzielle Lohnarbeiter? Warum sind Kriegsrentner und Paupers "Staatsagenten"(23)? Und schließlich werden gar die Rentner zur Mittelklasse

20 Marx und Engels drücken das schon in der „Heiligen Familie“ aus, wenn sie schreiben, daß auch die Kapitalisten von der Entfremdung betroffen sind, sich in ihr allerdings wohlfühlen.

21 Bd. II/S. 335

22 Bd. II, S. $272 \mathrm{ff}$.

23 Bd. II, S. 261 u. 373 
gezählt (24), wo sie doch nicht aus abgeleiteter Revenue, sondern aus eigener, ,,akkumulierter ${ }^{66}$ - wenn auch interpersonell und intertemporär umverteilt - leben. Viel schlüssiger wäre es, die Rentner je nach der Herkunft der Rente als passive Teile der entsprechenden Klasse zuzurechnen, wie es das PKA an anderer Stelle (25) auch tut.

Mit diesen Fragen haben wir uns selbst auf die Ebene der Zurechnungen begeben, und da liegt schon ein zentraler Fehler. Es geht nicht darum, das eine oder andere Gesellschaftsmitglied einer noch dazu unzureichend entwickelten abstrakten Bestimmung zuzuordnen. Es muß die Funktion der Gesellschaftsmitglieder in der ökonomischen und politischen Reproduktion der bürgerlichen Gesellschaft entwickelt werden, und zwar sowohl auf der Ebene der Kritik der politischen ơkonomie wie auf der Ebene ihrer widersprüchlichen Durchsetzung in der Geschichte. Erst dann lassen sich, auf der Basis der konkreten Arbeitsbedingungen und Bewußtseinsformen, politisch umsetzbare Klassenlinien schlüssig ziehen. Das Projekt Klassenanalyse ist zur Oberfläche der Gesellschaft gelangt, wie die Jungfrau zum Kind. Es hat sie schematisch geordnet, künstlich gruppiert und die innere gesellschaftliche Dynamik und konkrete Ausprägung der sozialstatistischen Klassengliederung konsequent ausgeblendet. Zwischen den begrifflichen Bestimmungen im ersten Band und den sozialstatistischen Mühen im zweiten liegt der Graben dieser konkreten Gesellschaft der BRD, nur mühsam von Rekursen und leeren Verallgemeinerungen überdeckt. Die Untersuchung des PKA ist also insoweit festzumachen als sozialstatistischer Ordnungsversuch der Oberfläche der bürgerlichen Gesellschaft in der BRD. Die in den Tiefen der Gesellschaft vor sich gehenden ökonomischen und politischen Prozesse, welche die Bedingung und Ursache ihrer Oberfläche und Veränderungen sind, bleiben unaufgedeckt. Behauptungen wie etwa die von der, ,Verlagerung von der materiellen zur geistigen Reproduktion der bürgerlichen Ordnung " (26) sagen daher nichts, als was jeder bewußte Zeitgenosse unmittelbar auch sehen könnte - abgesehen davon, daß es in dieser Form nicht stimmt.

\section{Revolutionäre Taktik des PKA (27)}

Hätten die Genossen auf S. 433 ihres Werkes geschwiegen, wären sie klug geblieben. Dann hätte ihre Analyse als sozialstatistischer Beitrag passieren können. Wenn wie wir umrissen zu haben hoffen - die vom PKA angestrebte Vermittlung vom System der Kritik der politischen Ökonomie und einem ,konkreten Stuick Zeitgeschichte" in klassenanalytischer Absicht letztlich in einer durch Auswälzung und Verdünnung des ,allgemeinen Begriffs“" gewonnenen deduktiven Aufschlüsselung

Bd. II, S. $42 \mathrm{f}$.

Bd. II, S. 441

Bd. II, S. 310 ff.

27

Zur folgenden Kritik vgl. Gerhard Armanski, Thesen zur „Revisionismus"kritik, Prokla $5 / 1972$ 
der Sozialstruktur endet, wenn die Fragen der konkreten Arbeits- und Lebensbedingungen und das Bewußtseins der Gesellschaftsmitglieder, der gewerkschaftlichen und politischen Organisierung, der politischen Bewegung überhaupt aus der Untersuchung fast vollkommen ausgeblendet sind, dann müssen die dessen ungeachtet entworfenen "Grundzüge revolutionärer Taktik in der BRD" auf tönernen Füßen stehen. Hier schlägt die falsche Methode um - in falsche Politik. Deren Grundlage ist dann auch nicht mehr die wirkliche Klassenbewegung, sondern hergeholte, ihr fremde, Setzungen.

„Der Kommunismus ist für uns nicht ein Zustand, der hergestellt werden soll, ein Ideal, wonach die Wirklichkeit sich zu richten haben wird. Wir nennen Kommunismus die wirkliche Bewegung, welche den jetzigen Zustand aufhebt. Die Bedingungen dieser Bewegung ergeben sich aus der jetzt bestehenden Voraussetzung $^{6}(28)$, „der bestimmten Konjunktur der Verhältnisse unter dem Bourgeoisregime" (29).

Wenn also diese „bestimmte Konjunktur“ nicht untersucht ist, können die den Zersetzungsprozeß der bürgerlichen Gesellschaft bewirkenden Kräfte materialistisch gar nicht angegeben werden. Wenn die geschichtlichen Ursachen des gegenwärtigen Arbeiterbewußtseins (Geschichte der Klassenkämpfe, Akkumulationsbewegung, Ungleichheiten und Fraktionierungen der Arbeiterklasse, Arbeits- und Lebensbedingungen, Organisation usw.) im Dunkeln bleiben, können Aussagen, welche diesen Zustand zusammenfassen und seine weitertreibenden Momente hervorheben, auch nicht ansatzweise getroffen werden. Dann muß man ans Ende jeder Untersuchung der einzelnen Fraktionen der Arbeiterklasse den ebenso schönen wie hilflosen Aufruf setzen, daß hier unbedingt vereinheitlicht werden muß (30), ohne sagen zu können, was die Bedingungen und Formen der Vereinheitlichung sind. Die „politische Apathie“ der Arbeiterklasse in der BRD (31) sei ,zusammenfassender Ausdruck für die in vielfältigen althergebrachten Traditionen verhaftete, mit wenig Selbstvertrauen in die eigene Stärke ausgestattete Arbeiterbewegung in der BRD ${ }^{\text {c }}$. Das erklärt nun allerdings überhaupt nichts, ist bloß tautologisch. Weil das Bewußtsein der Arbeiter so niedrig ist, ist die Arbeiterbewegung schwach. Weil die Arbeiterbewegung schwach ist, sind die Arbeiter ,politisch apathisch“. Sucht man weiter, findet man als Antwort auf den so gekennzeichneten Zustand lediglich den Verweis, daß die verschiedenen Gruppen innerhalb der Arbeiterklasse gegeneinander rangeln und sich noch nicht als Mitglieder einer Klasse begreifen. Die materialistische Analyse verflacht zur Sozialpsychologie. Warum die Arbeiter in der BRD so apathisch sind, warum sie sich in Gruppenkonflikten verzehren, wird dem neugierigen Leser nicht verraten. Und doch wäre die Antwort zentral für die Frage, welche politische Taktik zur Aufhebung dieses Zustandes einzuschlagen wäre. A.a.O., S. 204

PKA Bd. II/S. 146 ff.

31 Bd. II/S. 434 
An die Stelle der Aufnahme der wirklichen Probleme, vor welche sich die Arbeiterklasse in der BRD gestellt sieht (die vielfältigen materiellen Spaltungsursachen, u. a. der Unterschied von Hand- und Kopfarbeit, die wachsende Konkurrenz unter den Bedingungen von Massenentlassungen und zunehmendem Lohndruck, die sich verschlechternden Reproduktionsbedingungen, die politische Disziplinierung in Betrieb und Staat, die Politik der Gewerkschaftsspitzen und der SPD (32) usw.), tritt eine, auch als solche noch unzureichende, Oberflächenbeschreibung. An eine schlecht abstrakte Bewußtseinsbeschreibung wird eine ebensolche Vereinheitlichungstaktik gehängt, die mit einer revolutionären Zusammenfassung und Beschleunigung der wirklichen Bewegung der Auflösung der bürgerlichen Gesellschaft nichts mehr gemein hat. Aber die "Kenntnis der wirklichen Verhältnisse" wird wohl dem „Parteiprogramm zur Umwälzung der bürgerlichen Verhältnisse" (33) überlassen.

Als taktisches Grundprinzip, welches, ,aus den Verhältnissen in der BRD $^{66}$ (34) folge, wird die ,Aktionseinheit der Arbeiterklasse ${ }^{66}$ (35) vorgestellt ein wahrhaft neuer Gedanke! Deren wesentliche Bestimmungen erschöpfen sich wieder nur in der pausenlosen Aufforderung, sich zu vereinheitlichen. Bezüglich der Bauern findet sich nur ein Abzug der Marx'schen Analyse der französischen Parzellenbauern im 19. Jahrhundert, nicht aber der Ausdruck der BRD-Verhältnisse (36).

Völlig unklar wird es bei der Frage des Verhältnisses der Arbeiterklasse zum „lohnabhängigen“ Teil der Mittelklassen. Der noch im ersten Band relativ klar umrissene Mittelklassenbegriff verfluichtigt sich bei der konkreten Klassenbestimmung in nichts, womit die eingangs vorgetragene Hypothese des PKA, die moderne bürgerliche Gesellschaft zerfalle im wesentlichen in Arbeiterklasse und Kapitalistenklasse, seine Bestätigung findet. Allerdings eine Bestätigung, die letztlich auf einen Kreislauf der Theorie hinausläuft, welche die Wirklichkeit ausgeschlossen hat. Denn die wirkliche Klassenbewegung ist mitnichten durch die plötzliche Verflüchtigung der Mittelklasse geklärt. Das PKA geht da geschickt so vor, daß sie die ,der Arbeiterklasse nahestehenden Gruppen der lohnabhängigen Mittelklassen" (37) - womit die Beschäftigten der Staatsbetriebe in erster Linie gemeint sind - unvermittelt in die ,proletarische Bewegung" (38) stellt, von der sie nur noch materielle Privilegien trennten. Vergessen sind die seitenlangen Ausführungen im ersten Band über die Rolle des Staates, den Staatsfetisch, die materielle Lage und Bewußtsein der Staatsbeschäftigten entscheidend prägen. Oder gilt das für die Beschäftigten in den Staatsbetrieben nicht? Die reale gesellschaftliche Grundlage einer solchen Aktionseinheit

32 Hier findet sich nur der fromme Wunsch, daß sich die Arbeiterklasse von der SPD löse und die Bestimmung der linken SPD als Aktionseinheitspartner der Kommunisten. Vgl. Bd. II/S. 436

33 Bd. II/S. 435

34 ebenda

35 Bd. II/S. 436

$36 \mathrm{Bd}$. IV/S. $437 \mathrm{f}$.

$37 \mathrm{Bd} . \mathrm{II} / \mathrm{S} .442$

38 Bd. II/S. 443 
und ihre leitenden Forderungen sind denn auch gar nicht weiter angegeben, wenn man nicht bloße Aufklärung (Im Stile von: „Es kann ihnen gesagt werden . . ." ; 39) für sie nimmt.

Ein anderer Teil der ,lohnabhängigen Mittelklassenss taucht unter der Rubrik

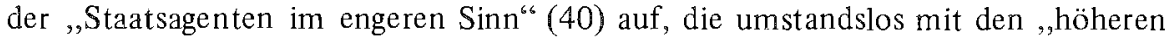
Staatsarbeitern" gleichgesetzt werden. Sie seien privilegiert, voller Klassenvorurteile, für die proletarische Bewegung nur teilweise zu gewinnen oder zu neutralisieren. ,Ihnen muß gesagt werden ${ }^{6}$, daß sie mit dem Sozialismus verschwinden - das ist alles. Der obwaltende Schematismus fällt nun auf das PKA selbst zurück. Denn von der Masse der nicht in Staatsbetrieben arbeitenden, aber auch nicht zu den ,höheren Staatsagenten“ zählenden (oder etwa doch?) staatlichen Lohnarbeitern im Gesundheits-, Sozial- und Erziehungswesen ist nicht die Rede. Also eine Personengruppe von ca. einer Million, die von den gegenwärtigen Verhältnissen hart angegriffen wird und teilweise in heftigen Abwehrkämpfen steht, verschwindet plötzlich aus der Analyse!

Wer beansprucht, Wichtiges zur revolutionären Taktik in der BRD beizutragen, darf sich solche Unterlassungen nicht erlauben. Denn immerhin bilden Lehrer, Sozialarbeiter, Krankenschwestern und Krankenhausärzte einen Hauptangriffspunkt der gegenwärtig laufenden Rationalisierung und Ökonomisierung im öffentlichen Dienst (40a). Immerhin sollen sie wesentlich zur Sanierung der Staatsfinanzen beitragen, indem sie gezwungen werden, bei sich verschlechternden Löhnen und Arbeitsbedingungen eine gleiche oder höhere Leistung zu erbringen. Und schließlich sind es gerade diese Gruppen der staatlichen Lohnarbeiter, die neben den Arbeitern der Staatsbetriebe einen Brennpunkt der sozialen Konflikte im öffentlichen Dienst bilden. Hier finden Kämpfe statt, die zur Auflösung von tradierten Bewußtseinsformen, vom Beamten- und Staatsfetisch praktisch tausendmal mehr beitragen, als das PKA überhaupt nur ahnt und wahrhaben will. Die Sache wird hier aber einfach so gelöst, daß sie getilgt wird. So grobmaschig und nichtssagend ist das klassenanalytische „Netz" des PKA geworden. Kein Wunder, denn bei Aufnahme jenes großen Teils der staatlichen Lohnarbeiter in der Erörterung der Taktik wäre man um die Mittelklassenfrage und damit das Bündnisproblem nicht herumgekommen. Wer von der Mittelklasse redet, muß auch von Bündnispolitik reden. Und das hätte wahrscheinlich der, ,allgemeinen Taktik in Ländern mit hochentwickeltem Kapitalverhältnis" widersprochen.

Die Lösung vieler Widersprüche wird der ,,selbständigen Arbeiterpartei mit bestimmtem Klassenprogramm ${ }^{66}$ zugewiesen, der ,,distinten Arbeiterpartei in der $\operatorname{BRD}^{66}(41)$, der $D K P$. Sie erscheint als deus ex machina, als konkreter Kern der

Bd. II/S. 445

40a Vgl, zur Rationalisierung und Ökonomisierung im öffentlichen Dienst und den Bedingungen und Formen der Kämpfe der staatlichen Lohnarbeiter: Gerhard Armanski, Boris Penth, Jörg Pohlmann, Staatsd iener im Klassenkampf, Erlangen 1975

Bd. II/S. 435 
eigenen verschwommenen Aussagen. Was die Aussagen dieses Kerns zur revolutionären Taktik der Kommunisten sind, wird leider nicht aufgenommen, nicht einmal gestreift. Von der Art und Weise, wie die DKP in die Analyse eingeführt wird, ist das auch gar nicht nötig. Sie ist ja auch nur abschließender und legitimierender Schlußstein des Gewölbes, keineswegs dessen theoretisch und praktisch entscheidender Träger. Wie die Taktik der Kommunisten bezüglich der Arbeiterklasse und den Mittelklassen nicht aus den realen Bedingungen und Kämpfen in der BRD entwickelt ist, so ist es auch die DKP nicht. Statt zu zeigen, welche Rolle die DKP in den Klassenkämpfen spielt, nimmt sie das PKA als bloße Zutat, mit Bestimmungen aus ganz anderen historischen Verhältnissen notdürtig abgestutzt (42), herein. Der Ring aus oberflächlicher Sozialstatistik, „unwirklicher" Taktik und ahistorischer politischer Dezision schließt sich.

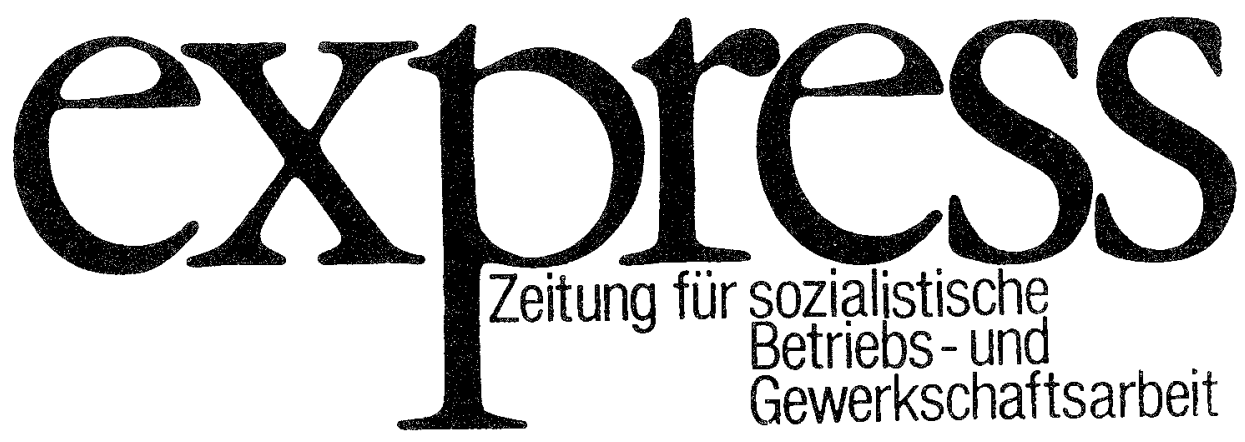

Sprachrohr der Kollegen und Genossen, die sozialistische Betriebsund Gewerkschaftsarbeit machen. Informationen über die Auseinandersetzung zwischen Kapital und Arbeit. Beiträge, die man nichł in den Gewerkschaftszeitungen findet.

Einzelpreis DM 1.-

Bezugspreis, jährlich, DM 14. - + 3.- Versandkosten

Probeexemplare anfordern bxw. Abonnemenfsbestellung bei
Verlag $2000 \mathrm{GmbH}, 605$ Offenbach 4, Postfach 591 .

42 Der Begriff der ,distinkten Arbeiterpartei" (vgl. Brief von Engels an F. A. Corge, 1886, in: Marx/Engels, Ausgewählte Briefe, Berlin (DDR), 1953, S. 470) stammt aus einer Periode unentwickelten Kapitalverhältnisses, aus dem 19. Jahrhundert, und meint zentral die Notwendigkeit, die Proletarier aus dem Schlepptau der bürgerlichen Parteien zu lösen. Wenn er für heute gelten soll, müßte zweierlei nachgewiesen werden: daß die SPD eine bürgerliche Partei ist; daß die DKP die ,,distinkte Arbeiterpartei" ist. 
Das folgende Schaubild I gehört zum folgenden Aufsatz, Seite 170!

ENTWICKLUNG DES PREISNIVEAUS, 1910-1914 =100

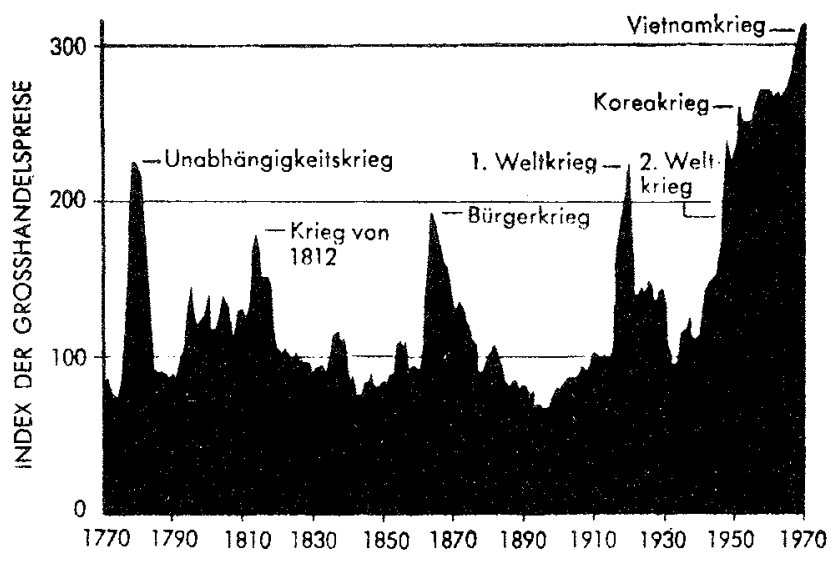

